\title{
Hepatocyte Growth Factor Effects on Mesenchymal Stem Cells Derived from Human Arteries: A Novel Strategy to Accelerate Vascular Ulcer Wound Healing
}

\author{
Sabrina Valente, ${ }^{1}$ Carmen Ciavarella, ${ }^{1}$ Emanuela Pasanisi, ${ }^{1}$ Francesca Ricci, ${ }^{2}$ \\ Andrea Stella, ${ }^{1}$ and Gianandrea Pasquinelli ${ }^{1}$ \\ ${ }^{1}$ Department of Experimental, Diagnostic and Specialty Medicine (DIMES), University of Bologna, \\ Via Massarenti 9, 40138 Bologna, Italy \\ ${ }^{2}$ Cardiovascular Tissue Bank-Immunohematology and Transfusion Medicine, University Hospital St. Orsola-Malpighi, \\ Polyclinic of Bologna, Via Massarenti 9, 40138 Bologna, Italy
}

Correspondence should be addressed to Sabrina Valente; sab.valente@gmail.com

Received 20 April 2015; Revised 11 August 2015; Accepted 23 August 2015

Academic Editor: Tao-Sheng Li

Copyright (c) 2016 Sabrina Valente et al. This is an open access article distributed under the Creative Commons Attribution License, which permits unrestricted use, distribution, and reproduction in any medium, provided the original work is properly cited.

\begin{abstract}
Vascular ulcers are a serious complication of peripheral vascular disease, especially in diabetics. Several approaches to treat the wounds are proposed but they show poor outcomes and require long healing times. Hepatocyte Growth Factor/Scatter Factor (HGF/SF) is a pleiotropic cytokine exerting many biological activities through the c-Met receptor. This study was aimed at verifying whether HGF/SF influences proliferation, migration, and angiogenesis on mesenchymal stem cells isolated from human arteries (hVW-MSCs). hVW-MSCs were exposed to NIBSC HGF/SF $(2.5,5,10$, and $70 \mathrm{ng} / \mathrm{mL})$ from 6 hrs to 7 days. HGF and c-MET mRNA and protein expression, cell proliferation (Alamar Blue and Ki-67 assay), migration (scratch and transwell assays), and angiogenesis (Matrigel) were investigated. hVW-MSCs displayed stemness features and expressed HGF and c-MET. HGF/SF did not increase hVW-MSC proliferation, whereas it enhanced the cell migration, the formation of capillary-like structures, and the expression of angiogenic markers (vWF, CD31, and KDR). The HGF/SF effects on hVW-MSC migration and angiogenic potential are of great interest to accelerate wound healing process. Local delivery of HGF/SF could therefore improve the healing of unresponsive vascular ulcers.
\end{abstract}

\section{Introduction}

The lower limb ulceration has prevalence of about $3 \%$ in the adult population over 65 years [1]. Foot ulcers mainly affect the arterial system and are particularly severe and devastating among diabetic patients, where they tend to become chronic. For this reason, foot ulcers significantly affect the expectancy and quality life of such patients, as well as healthcare expenditures. Arterial ulcers are a consequence of diffuse atherosclerotic narrowing of the leg arteries resulting in a significant reduction of blood flow to the lower limb. The main therapy consists of restoring blood flow by angioplasty or bypass and the removal of the debridement and the necrotic tissue to accelerate the wound healing. Additional treatments have been recently introduced, such as biological dressing, physical therapy (hyperbaric oxygen and negative pressure therapy), and compression therapy [2]; however, clinical results are still less than optimal.

To date, surgical revascularization remains the milestone of any arterial ulcer treatment; however, blood flow restoration should be associated with treatments aimed at reactivating ineffective autologous healing processes, such as inflammatory cell responses and local angiogenesis whose ineffectiveness is responsible for incomplete and delayed wound healing. In this respect, the use of gene and cell therapies [3] and natural or synthetic engineered matrices $[2,4]$ has gained interest in the scientific community; as an example, interesting results on the complete wound healing were seen after transplantation of biomimetic tissue engineered dermis substitutes with allogeneic keratinocytes and fibroblasts cells inside ulcerated tissue $[5,6]$. 
Interestingly, both animal and human studies advocate the adult mesenchymal stem cells derived from bone marrow $[7,8]$ as well as adipose tissue [9-11] as ideal candidates to treat nonhealing wound due to their ability to differentiate in multiple mesengenic lineages and their capability to facilitate angiogenesis through the secretion of proangiogenic growth factors, even if their exact contribution to wound healing has not been completely understood.

Human derived autologous platelet-rich plasma (PRP) is also a promising wound healing treatment, due to the mitogenic and chemoattracting effects exerted by growth factors released at high concentrations after platelet $\alpha$-granules degranulation [12]. Some case reports and few clinical trials [13-15] showed positive effects of PRP on reepithelialization of nonhealing wounds.

Alternatively, single or combined growth factors including Platelet-Derived Growth Factor (PDGF), Vascular Endothelial Growth Factor (VEGF), Transforming Growth Factor-beta (TGB-beta), Fibroblast Growth Factor (FGF), Epidermal Growth Factor (EGF), and Granulocyte Macrophage-Colony Stimulating Factor (GM-CSF) are largely studied $[16,17]$, thanks to their multiple effects on wound healing including cell proliferation and mobilization, extracellular matrix production, and angiogenesis even though different outcomes are reported. In addition, the Hepatocyte Growth Factor/Scatter Factor (HGF/SF) is a multifunctional cytokine involved in numerous biological responses including cell proliferation/survival, angiogenesis, morphogenesis, and motogenesis [18] as well as inflammation and fibrosis inhibition [19]; these actions are exerted through its tyrosine kinase receptor, c-Met [20], that is primarily expressed in epithelial cells; however, few reports indicate that adult mesenchymal stem cells also express c-Met [21-23].

In this study, we tested the in vitro effects of HGF/SF on a multipotent mesenchymal stem cells population that our research group isolated from the vascular wall of adult human arteries (hVW-MSCs) [24]. The hVW-MSC exposure to $\mathrm{HGF} / \mathrm{SF}$ could represent a strategy to improve and accelerate the wound healing process in foot ulcers. In particular, we investigated the HGF/SF effect on cell proliferation and viability using Alamar Blue assay and Ki-67 immunofluorescence staining, cell migration and motility abilities through scratch and transwell assays, and angiogenic potential to form capillary-like structures in a Matrigel assay.

\section{Materials and Methods}

2.1. Human Vascular Wall-Mesenchymal Stem Cell Isolation and Culture Condition. Vascular wall-mesenchymal stem cells (hVW-MSCs) isolated from human arteries were used according to the ethic protocol (APP-13-01) approved by the Local Ethics Committee of University Hospital St. OrsolaMalpighi of Bologna in Italy and with consent informed. Their isolation, characterization, and stemness investigation were performed using methods described elsewhere [24]. Briefly, the human arteries were enzymatically digested overnight at $37^{\circ} \mathrm{C}$ in a rotor apparatus using serum-free DMEM culture medium supplemented with $0.3 \mathrm{mg} / \mathrm{mL}$
Liberase type II (Roche, Milan, Italy) followed by filtration through 40-70-100 $\mu \mathrm{m}$ nylon mesh cell strainer (Becton Dickinson; Franklin Lakes, NJ) seeded on collagen-coated flasks and cultured in complete DMEM plus 20\% Fetal Bovine Serum (FBS) at $37^{\circ} \mathrm{C}$ in a humidified atmosphere of $5 \% \mathrm{CO}_{2}$ for 3 days. After floating cells removal, adherent hVW-MSCs were expanded until confluence replacing the cell culture with fresh medium every 2-3 days. Immunophenotype, stemness features, and multilineage potential were investigated confirming their mesenchymal identity [24]. In this study, experiments were performed using cells taken at passages 3 and 4 and cultured in DMEM plus $10 \%$ of FBS with or without Hepatocyte Growth Factor/Scatter Factor (HGF/SF) (WHO Reference Reagent, HGF/SF, NIBSC code: 96/564, National Institute for Biological Standards and Control, Potter Bar, Hertfordshire, ENG 3QG) at different time (from $6 \mathrm{hrs}$ to 7 days) and concentrations (from 2.5 to $70 \mathrm{ng} / \mathrm{mL}$ ). Cell starvation with low percentage of serum ( $0.5 \%$ FBS $)$ for $12 \mathrm{hrs}$ was performed to induce cell cycle synchronization before pretreatment with 0.2 $\mu \mathrm{M}$ PHA-665752 inhibitor (Tocris Bioscience, Bristol, UK) for $12 \mathrm{hrs,} \mathrm{followed} \mathrm{by} \mathrm{HGF/SF} \mathrm{addition.}$ All experiments were executed in triplicate.

2.2. RNA Extraction and RT-PCR. RT-PCR was performed on hVW-MSCs to detect the basal expression of HGF and its receptor c-Met. Total RNA was extracted from hVW-MSCs using TRIreagent according to the manufacturer's instructions (TRIzol reagent; Invitrogen). Reverse transcription of $1 \mu \mathrm{g}$ of total RNA was carried out in a $20 \mu \mathrm{L}$ volume of reaction using a High Capacity Reverse Transcription Kit (Applied Biosystems, Carlsbad, CA, USA). Polymerase Chain Reaction (PCR) products were analyzed by electrophoresis on a $2 \%$ agarose gel, stained with ethidium bromide and photographed under ultraviolet light. All PCR product sizes were identified loading a 100-base-pair (bp) DNA ladder and normalized to GAPDH, used as housekeeping gene. The PCR primers were purchased from Sigma-Aldrich. Genes and respective primers are presented in Table 1.

2.3. Western Blot Analysis. Total cellular proteins were extracted by untreated and treated hVW-MSCs using lysis buffer (KH2PO4 0.1 M pH 7.5, NP-40 1\%, and $0.1 \mathrm{mM} \alpha$ glycerolphosphate, added with complete protease inhibitors cocktail, Roche Diagnostics) and quantified spectrophometrically with the Bio-Rad Protein Assay (Bio-Rad Laboratories, Hempstead, UK). Thirty $\mu$ g proteins were subjected to $8 \%$ SDS-PAGE and transferred to nitrocellulose membrane (GE Healthcare Life Sciences, Amersham) at $30 \mathrm{~mA}$ for $2 \mathrm{hrs}$ and 30 minutes. The membrane was blocked with 5\% nonfat dry milk in TBS-tween for 1 hour at room temperature (RT), incubated with primary antibodies against c-Met $(1: 500$, Santa Cruz Biotechnologies), HGF (1:500, Santa Cruz Biotechnologies), and anti- $\alpha$-actin (clone AC-74, SigmaAldrich) at $4^{\circ} \mathrm{C}$ overnight. Secondary antibodies (human anti-rabbit/mouse horseradish peroxidase-conjugated (GE Healthcare, Milan, Italy)) were used at 1:10000 dilutions for $1 \mathrm{~h}$ at room temperature (RT). Protein signal was detected using Westar $\eta \mathrm{C}$ chemiluminescent substrate (Cyanagen). 
TABLE 1: List of primers used for RT-PCR in hVW-MSCs.

\begin{tabular}{|c|c|c|c|}
\hline Gene & Primer sequence & Product size (bp) & $\mathrm{T}\left({ }^{\circ} \mathrm{C}\right)$ \\
\hline \multirow{2}{*}{ GAPDH } & FWD $5^{\prime}$-ACCACAGTCCATGCCATCAC- $3^{\prime}$ & \multirow{2}{*}{452} & \multirow{2}{*}{61} \\
\hline & REV 5'-TCCACCACCCTGTTGCTGTA-3' & & \\
\hline \multirow{2}{*}{ c-MET } & FWD 5'-AGAAATTCATCAGGCTGTGAAGCGCG-3' & \multirow{2}{*}{440} & \multirow{2}{*}{68} \\
\hline & REV 5' -TTCCTCCGATCGCACACATTTGTCG-3' & & \\
\hline \multirow{2}{*}{ HGF } & FWD 5'-TTTGCCTTCGAGCTATCGGG-3' & \multirow{2}{*}{254} & \multirow{2}{*}{62} \\
\hline & REV 5'-GCAAGAATTTGTGCCGGTGT-3' & & \\
\hline
\end{tabular}

2.4. Cell Viability and Proliferation Evaluation. Cell viability was investigated using Alamar Blue fluorescence assay (Invitrogen, Milan, Italy). The hVW-MSCs were seeded in a 12-multiwell plate at the density of $3 \times 10^{4}$ and cultured in complete DMEM with or without HGF/SF $(2.5,5$, and $10 \mathrm{ng} / \mathrm{mL})$ for 1,3 , and 7 days. Alamar Blue solution $(10 \%$ $\mathrm{v} / \mathrm{v}$ in cultured medium) was added to each well at the end of treatments and incubated for $4 \mathrm{hrs}$ at $37^{\circ} \mathrm{C}$ according to the manufacturer's instruction. Alamar Blue fluorescence $(\mathrm{Ex} / \mathrm{Em}=540 / 590 \mathrm{~nm})$ of three replicates in each well was measured in a Wallac VICTOR2 multiplate reader (Perkin Elmer, Milan, Italy). In addition, a standard polystyrene well was used to measure the background fluorescence; this fluorescence was subtracted from the reading of each well.

Cell proliferation was assessed using single immunofluorescence staining for cycling cells expressing Ki-67 protein. hVW-MSCs were plated at a density of $6 \times 10^{5}$ on coverslip in 6 -well plates in DMEM overnight to allow the cell confluence and treated with or without $\operatorname{HGF} / \mathrm{SF}(2.5,5,10$, and $70 \mathrm{ng} / \mathrm{mL})$ for 6 and $24 \mathrm{hrs}$. In parallel experiments, additional cellseeded glass was starved in $0.5 \%$ FBS for $12 \mathrm{hrs}$, pretreated with $0.2 \mu \mathrm{M}$ PHA-665752 inhibitor, and subjected to HGF/SF exposure at the same time and concentrations. Untreated cells were used as a control.

2.5. Immunofluorescence Staining. At the end of treatments, cells were washed, fixed, and permeabilized in $2 \%$ paraformaldehyde in PBS with 1\% Tryton X-100 for 4 minutes at RT, blocked with $1 \%$ bovine serum albumin (BSA) for 30 minutes at RT to reduce nonspecific staining, and labeled with monoclonal antibody against nuclear transcription factor Ki-67 (1:100, Novocastra, Leica Microsystems, Wetzlar, Germany) and intermediate filament Vimentin (1:100, Dako Cytomation, Glostrup, Denmark). Samples were washed, stained with AlexaFluor-488 (1:250, Life Technology, Carlsbad, CA, USA) secondary antibody in the dark, and counterstained with Pro Long antifade reagent with DAPI (Molecular Probes, Milan, Italy). All incubations were performed for $1 \mathrm{hr}$ at $37^{\circ} \mathrm{C}$ in a wet chamber; both antibodies were diluted in $1 \%$ BSA in PBS. Samples were observed and photographed in a Leica DMI6000 B inverted fluorescence microscope (Leica Micro-systems, Wetzlar, Germany). Negative control was performed by omitting the primary antibody and no fluorescence was detected. For each experimental condition, the number of Ki-67 intensely stained cells as well as DAPI stained nuclei was manually counted on ten random fields and their values were expressed in percentage as ratio of Ki-67 stained cells on total cells number.
2.6. In Vitro Wound Healing Assay. Cell migration was investigated using a scratch assay. HVW-MSCs were seeded in 12-multiwell plate at a density of $1 \times 10^{5} /$ well and grown until to confluence. Cell monolayers were wounded with a p200 pipette tip, washed with PBS to remove cell debris, and treated with complete DMEM containing $\operatorname{HGF} / \mathrm{SF}(2.5,5,10$, and $70 \mathrm{ng} / \mathrm{mL}$ ) for $24 \mathrm{hrs}$. In further experiments, cells were starved, inhibited with $0.2 \mu \mathrm{M}$ PHA-665752, and cultured in presence of $\mathrm{HGF} / \mathrm{SF}$ at the same time and concentrations. At the end of HGF/SF treatment for both experiments, cells were fixed in absolute methanol for 10 minutes, washed in PBS, stained with $0.1 \%$ Crystal Violet in 25\% methanol for 30 minutes, and air-dried; all steps were performed at RT. The wound closure was observed under a phase-contrast light microscope (LM) equipped with a digital camera (Nikon), acquiring images for each sample at time 0 and $24 \mathrm{hrs}$, respectively, using Software NIS-elements D3.2 Nikon (Tokyo, Japan). Computer-assisted image analysis (Image-Pro Plus software, Media Cybernetics, http://www.mediacy.com/) was employed to perform the quantification of the area of cells migrated into scratched area as well as the total wounded area in three different fields. Values were expressed in percentage as ratio of migrated cell area on total scratched area. Additional cell-seeded glasses were used for Vimentin intermediate filaments immunofluorescence staining.

2.7. Transwell Migration Assay. The cellular ability to migrate through a porous membrane under the influence of a chemoattractant factor was evaluated using transwell chambers (Costar, Corning Incorporated, NY, USA). Briefly, $500 \mu \mathrm{L}$ of DMEM with $10 \%$ FBS with or without chemoattractant HGF/SF $(10 \mathrm{ng} / \mathrm{mL})$ was placed below the polycarbonate membrane with $8 \mu \mathrm{m}$ pores while $300 \mu \mathrm{L}$ of cellular suspension containing $2.5 \times 10^{4} \mathrm{hVW}$-MSCs in growth medium was plated on the upper layer of the membrane for $24 \mathrm{hrs}$ at $37^{\circ} \mathrm{C}$ in incubator. After the manual removal of the nonmigrated cells from the upper side of the membranes, the inserts were detached from the plastic support using a scalpel, fixed, stained with $0.1 \%$ Crystal Violet for 30 minutes at RT, and mounted on glass slide. The cells migrated in the lower layer were observed using a Leitz Diaplan LM (Wetzlar, Germany) equipped with a video camera (JVC 3CCD video camera, KY-F55B, Jokohama, Japan). Digital images were acquired at $10 \mathrm{x}$ of magnification using Image-Pro Plus 6 software (Media Cybernetics). Additional membranes were processed for SEM analysis.

2.8. Scanning Electron Microscopy (SEM). For SEM, samples were rinsed in $0.15 \mathrm{M}$ phosphate buffer to remove 
the culture medium, fixed in $2.5 \%$ buffered glutaraldehyde (TAAB Laboratories, UK) overnight at $4^{\circ} \mathrm{C}$, washed again in phosphate buffer, postfixed in $1 \%$ osmium tetroxide in $0.1 \mathrm{M}$ phosphate buffer, wet in distilled water, and dehydrated with increasing ethanol concentrations (70-100\%). Each step was performed at RT for 15 minutes. For drying the samples, they were immersed before in a solution of $50 \%$ absolute ethanol $/ 50 \%$ hexamethyldisilazane (HMDS, Fluka Analytical, Sigma, Steinheim, Germany) and after in pure HMDS for 30 minutes each passage at RT and finally airdried. Before observation, the samples were mounted on aluminum supports (Multilab type stub pin 1/2, Surrey, UK) using a silver paste maintaining the cell-seeding surface, coated with gold in a sputtering device (Quorum Q150RS, Technologies Ltd., Laughton, UK), and observed at 5-10 kV with a Quanta 250 (FEI Company, Milan, Italy) scanning electron microscope.

2.9. In Vitro Tube Formation Assay. Angiogenic potential to form capillary-like tubes was measured using a semisolid matrix after culturing confluent hVW-MSCs for 7 days in DMEM containing 2\% FBS with $10 \mathrm{ng} / \mathrm{mL} \mathrm{HGF} / \mathrm{SF}$ or $50 \mathrm{ng} / \mathrm{mL}$ VEGF (Sigma); control cells were maintained in basal medium plus 10\% FBS. At the end of treatments, a 96-well culture plate was coated with $50 \mu \mathrm{L}$ of Matrigel (BD Bioscence) solution for $1 \mathrm{hr}$ at $37^{\circ} \mathrm{C}$. After that, untreated and treated cell suspensions were placed onto the solidified layer of Matrigel at the density of $15 \times 10^{3} \mathrm{hVW}$-MSCs for well. Human Umbilical Vein Endothelial Cells (HUVEC) were used as a positive control. Tubular blood vessels-like structures were observed under inverted LM and documented with a digital camera (Nikon) after 2, 6, and $24 \mathrm{hrs}$. To quantify in vitro angiogenesis, the number of capillarylike structures was manually counted on digitalized images taken at $4 \mathrm{x}$ magnification for each experimental condition. In parallel experiments, flow cytometry was performed to detect the expression of vWF, KDR, and CD31 mature endothelial cell markers in control as well as growth factors-treated cells. For surface antigen, the treated cells were rinsed in PBS, labeled with primary antibodies against KDR-APC and CD31-PE. To reveal vWF expression, the cells were fixed and permeabilized with the IntraPep Kit (Beckman-Coulter), incubated with von Willebrand Factor (vWF; Santa Cruz Biotechnology, Santa Cruz, CA, USA), and subsequently incubated with anti-mouse IgG-FITC (Dako) secondary antibody. The endothelial lineage commitment was quantified in all experimental conditions attributing a score from 0 to 4 according to the flow cytometry values of each mature endothelial cell marker. Final values are reported as mean.

2.10. Statistical Analysis. Results were expressed as the means \pm SEM. GraphPad Prism 5.0 software (GraphPad Prism software, San Diego, CA) was used to perform statistical analysis and to create graphical representations. Statistical differences between samples were determinated using unpaired Student's $t$-test and one-way ANOVA test for comparison between more than two groups, followed by Bonferroni posttest; $p$ value $<0.05$ was considered to be statistically significant.

\section{Results}

3.1. $h V W$-MSC Isolation and Stemness Property. hVW-MSCs isolated from human arteries showed a spindle-shaped morphology and a marked adhesion growth (Figure 1(a)). hVWMSCs expressed mesenchymal (CD44, CD73, CD90, CD105, HLA-G), stemness (Stro-1, Oct-4, and Notch-1), pericyte (CD146, PDGFR- $\beta$, and NG2), and neuronal (nestin) markers (Figure 1(b)), together with the plasticity to differentiate in multiple mesengenic lineages, clonogenicity, and immunomodulatory functions; details on their morphology, immunophenotype, and molecular and functional features are reported elsewhere [24].

3.2. HGF and c-MET $m R N A$ and Protein Expression in $h V W$ MSCs. In a previous study, Neuss et al. demonstrated that hMSCs derived from bone marrow expressed HGF and its receptor, c-Met [21]. In this study we verified whether hVWMSCs, recovered from human arteries, also may constitutively possess these genes. Gene expression analysis showed that hVW-MSCs express HGF and $c-M E T$ receptor mRNA (Figure 1(c)). Western Blot confirmed the expression of the c-Met receptor in hVW-MSCs and showed increased protein levels in hVW-MSCs exposed to HGF/SF, especially at $10 \mathrm{ng} / \mathrm{mL}$ (24 hrs). Meanwhile, the expression of the HGF protein did not show evident differences under HGF/SF stimulation (Figure 1(d)).

3.3. HGF/SF Effect on $h V W$-MSC Proliferation. hVW-MSCs were exposed to $2.5,5$, and $10 \mathrm{ng} / \mathrm{mL}$ of $\mathrm{HGF} / \mathrm{SF}$ for a wide range of time (from 6 hrs to 7 days). From day 1 to day 3, Alamar Blue fluorescence assay revealed an increased cell viability in all experimental conditions including control that was related to overall cell population doubling; no difference between untreated and HGF/SF-treated hVW-MSCs was seen at day 3 and day 7 (Figure 2(a)). The cell proliferation following $6 \mathrm{hrs}$ and $24 \mathrm{hrs}$ of exposure to 2.5, 5, 10, and $70 \mathrm{ng} / \mathrm{mL} \mathrm{HGF} / \mathrm{SF}$ was assayed by Ki-67 staining. Immunostaining analysis on hVW-MSCs exposed to HGF/SF for $6 \mathrm{hrs}$ showed an increased Ki-67 expression in a dose-dependent manner, in comparison to the untreated hVW-MSCs; the percentage of Ki-67 positive cells was significantly higher when hVW-MSCs were exposed to $\mathrm{HGF} / \mathrm{SF}$ at $10 \mathrm{ng} / \mathrm{mL}$ $(29.6 \pm 4.1$ in HGF/SF-treated hVW-MSCs versus $19.4 \pm$ 4.8 in untreated controls, $p<0.05$, one-way ANOVA test followed by Bonferroni posttest). Conversely, after $24 \mathrm{hrs}$ of incubation, the percentage of cycling cells positive to Ki-67 was significantly decreased in all the concentrations tested $(30.3 \pm 5.6$ in $2.5 \mathrm{ng} / \mathrm{mL}, 28.4 \pm 1.5 \mathrm{in} 5 \mathrm{ng} / \mathrm{mL}$, $28.4 \pm 4.3 \mathrm{in} 10 \mathrm{ng} / \mathrm{mL}$, and $26.5 \pm 4.5 \mathrm{in} 70 \mathrm{ng} / \mathrm{mL}$, versus $39.9 \pm 4.0$ in control cells; $p<0.05$; one-way ANOVA test followed by Bonferroni posttest) (Figure 2(b)). hVWMSCs exposed to PHA-665752 inhibitor showed a significant decrease of Ki-67 expression levels (12.7 \pm 5.9 versus $39.9 \pm$ 4.0 unexposed hVW-MSCs, $p<0.05$, one-way ANOVA test followed by Bonferroni posttest). The HGF/SF addition to PHA-665752-pretreated hVW-MSCs restored the hVWMSCs proliferation and the percentage of Ki-67 positive cells 

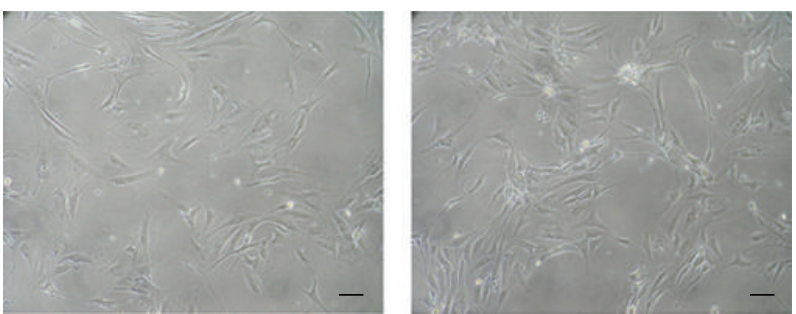

(a)

\begin{tabular}{llll}
\hline Mesenchymal markers & Stemness markers & Pericyte markers & Hematopoietic and vascular markers \\
\hline CD44 (\%) 99.8 & Stro-1 (\%) 73.4 & CD146 (\%) 12.2 & CD31 (\%) 1.5 \\
CD90 (\%) 90.8 & Oct-4 (\%) 36.4 & NG2 (\%) 33.0 & CD34 (\%) 6.8 \\
CD105 (\%) 99.6 & Notch-1 (\%) 33.0 & PDGF-rbeta (\%) 98.4 & CD45 (\%) 0.3 \\
\hline
\end{tabular}

(b)

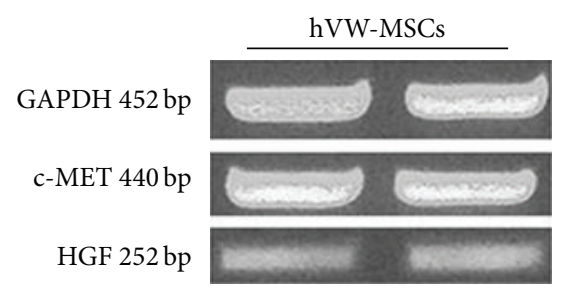

(c)

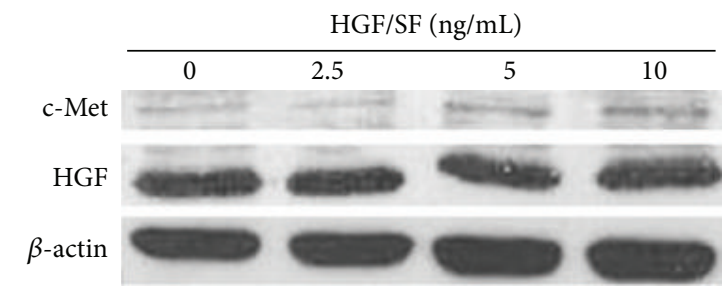

(d)

FIGURE 1: hVW-MSC aspect and HGF/c-MET expression. (a) hVW-MSCs were enzymatically isolated from human arteries and, at passage 3 , they exhibited adherence to the plastic substrate and a typical spindle-shaped morphology. Scale bars $=100 \mu \mathrm{m}$. (b) Flow cytometry analysis of mesenchymal, stemness, pericyte, and hematopoietic and vascular markers expressed in our cell model of hVW-MSCs. Values are reported in percentage of positivity. (c) Basal expression of HGF (252 bp) and c-MET (440 bp) mRNA in hVW-MSCs, detected by RT-PCR. (d) c-Met and HGF protein detection on cell lysates of hVW-MSCs exposed to HGF/SF (0, 2.5, 5, and $10 \mathrm{ng} / \mathrm{mL})$ for $24 \mathrm{hrs}$.

revealed overlapping results with those obtained without PHA-665752 (Figure 2(c)). Results with c-Met inhibition definitively confirmed that HGF/SF does not increase the hVW-MSCs proliferation.

\subsection{HGF/SF Effect on $h V W$-MSC Migration. To investigate} the in vitro HGF/SF effect on cell migration, we used a wounded confluent hVW-MSC monolayer model cultured with or without HGF/SF for $24 \mathrm{hrs}$. hVW-MSCs exposed to $\operatorname{HGF} / \mathrm{SF}(2.5,5,10$, and $70 \mathrm{ng} / \mathrm{mL})$ covered the wounded area more efficiently than untreated controls. The cell migration was reduced by adding $0.2 \mu \mathrm{M}$ of the c-Met inhibitor, PHA-665752, in the culture medium before any HGF/SF treatment. Moreover, in absence of HGF/SF stimuli, hVWMSCs exhibited a spontaneous capacity to move into the cell-free wounded area; the spontaneous migration was probably stimulated by an autocrine mechanism related to endogenous HGF release. The addition of HGF/SF to PHA-pretreated hVW-MSCs did not completely restore the cell migration (Figures 3(a) and 3(b)). To corroborate light microscopy results, SEM investigation was performed at the same doses and time of HGF/SF treatment. SEM confirmed the high efficacy of HGF/SF to promote hVW-MSC migration into scratched area restoring the cell monolayer scratched by p200 pipette tip when compared to control cells (data not show); similar results were seen using single immunofluorescence staining for Vimentin. Notably, while Vimentin was expressed in all hVW-MSCs, the labeling fluorescence intensity was more intense in the cells migrated in the wound area; this fluorescence pattern was interpreted as a feature of cytoskeleton remodeling (Figure 3(c)).

3.5. HGF/SF Effect on hVW-MSC Motility. The HGF/SF ability to mobilize and chemoattract hVW-MSCs was tested through a transwell migration assay. Crystal Violet dye showed that HGF/SF (10 ng/mL) enhanced hWV-MSC motility when compared to spontaneous hVW-MSC migration. At SEM, the majority of hVW-MSCs remained on the seeding surface in absence of HGF/SF; on the contrary, hVWMSCs efficiently colonized the migration surface after adding $10 \mathrm{ng} / \mathrm{mL}$ of HGF/SF to the lower compartment (Figures 4(a) and $4(\mathrm{~b}))$.

3.6. HGF/SF Effect on $h V W-M S C$ Angiogenic Potential. The $\mathrm{hVW}-\mathrm{MSC}$ angiogenic differentiation under the presence of $\mathrm{HGF} / \mathrm{SF}$ was assessed in a $3 \mathrm{D}$ semisolid matrix assay. HGF/SF $(10 \mathrm{ng} / \mathrm{mL})$ or VEGF $(50 \mathrm{ng} / \mathrm{mL})$ induced the formation of capillary-like structures that peaked at $6 \mathrm{hrs}$ and persisted until $24 \mathrm{hrs}$. After $2 \mathrm{hrs}$ of exposure to HGF/SF or VEGF, hVW-MSCs aligned and branched from the cell periphery to form tube-like structures. At $6 \mathrm{hrs}$, the density of the HGF/SF or VEGF-induced capillary-like network 


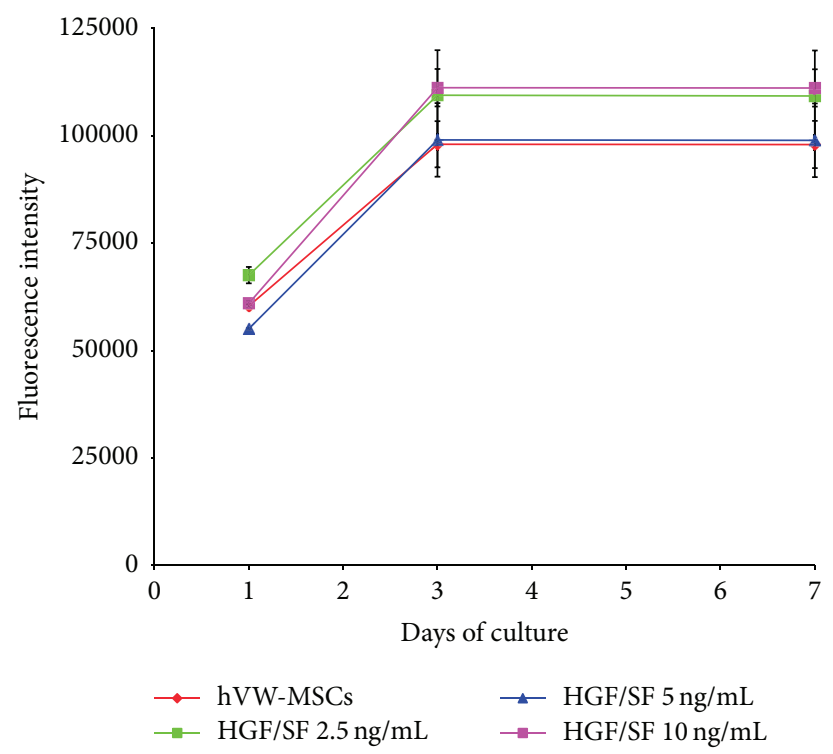

(a)

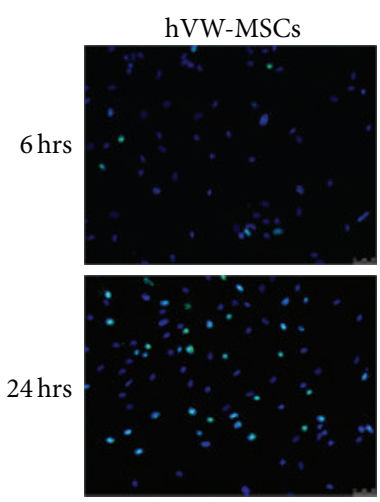

hVW-MSCs + HGF/SF
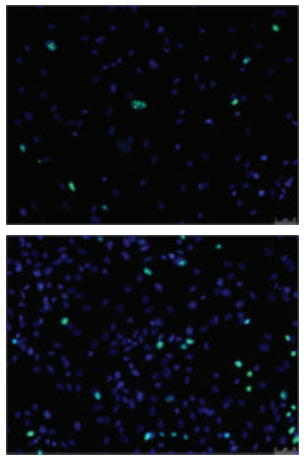

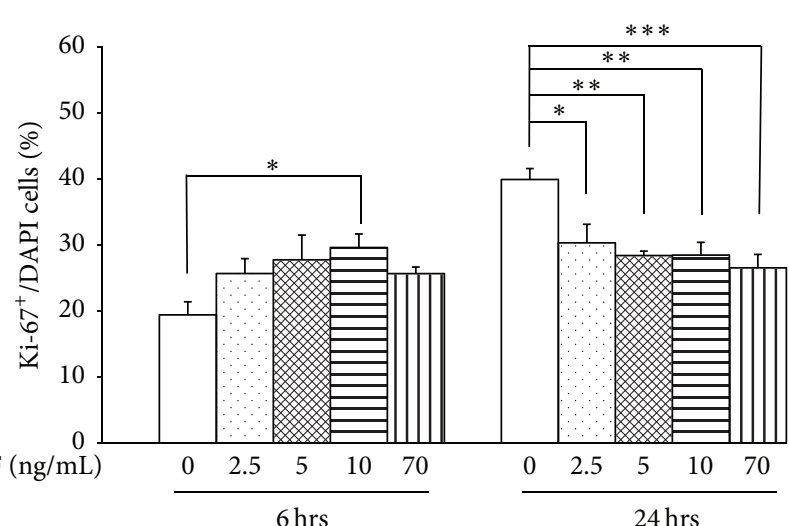

(b)
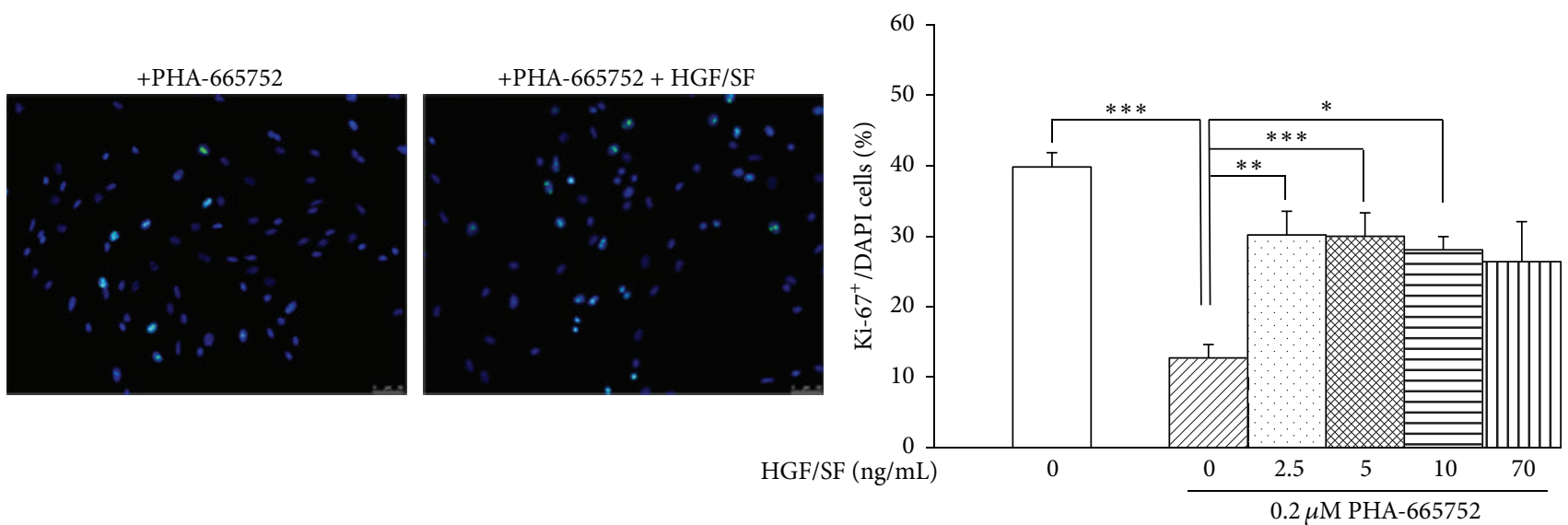

(c)

Figure 2: Proliferative effect of HGF/SF on hVW-MSCs. (a) In vitro proliferation of hVW-MSCs exposed to HGF/SF assessed by Alamar Blue fluorescence assay up to 7 days. From day 1 to day 3, the fluorescence intensity was increased in HGF/SF-treated hVW-MSCs including untreated cells (red) while no difference was seen between hVW-MSCs stimulated by HGF/SF and untreated cells at 3 and 7 days. (b) Representative images of cycling cells expressing Ki-67 protein after 6 and 24 hrs of incubation with HGF/SF, compared to controls (hVWMSCs). Ki-67 positive cells (green) and DAPI (nuclei in blue). After 6 hrs, the HGF/SF stimulation induced a slight increase of Ki-67 positive $\mathrm{hVW}$-MSCs percentage on total DAPI cells; after $24 \mathrm{hrs}$ of HGF/SF exposure, the percentage of Ki-67 positive cells is approximately reduced of the $15 \%$ in all the experimental conditions. Scale bars $=50 \mu \mathrm{m}$. (c) Images and quantitative analysis of hVW-MSCs cultured with PHA- 665752 and then exposed to HGF/SF. c-Met inhibitor before HGF/SF incubation revealed almost similar Ki-67 percentage reduced in comparison to control cells. Scale bars $=50 \mu \mathrm{m}$. (b)-(c) ${ }^{*},{ }^{* *},{ }^{* * *} p$ value $<0.05$, and one-way ANOVA test followed by Bonferroni posttest. 

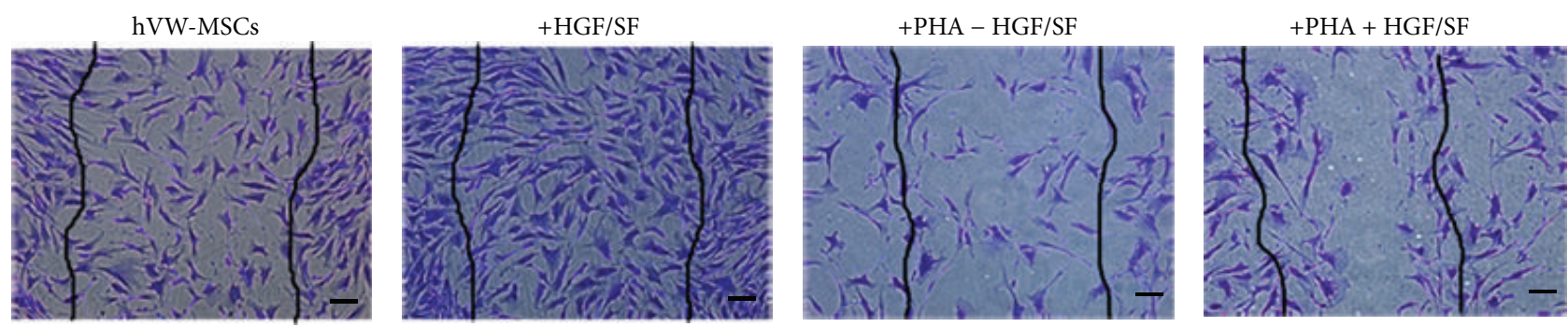

(a)

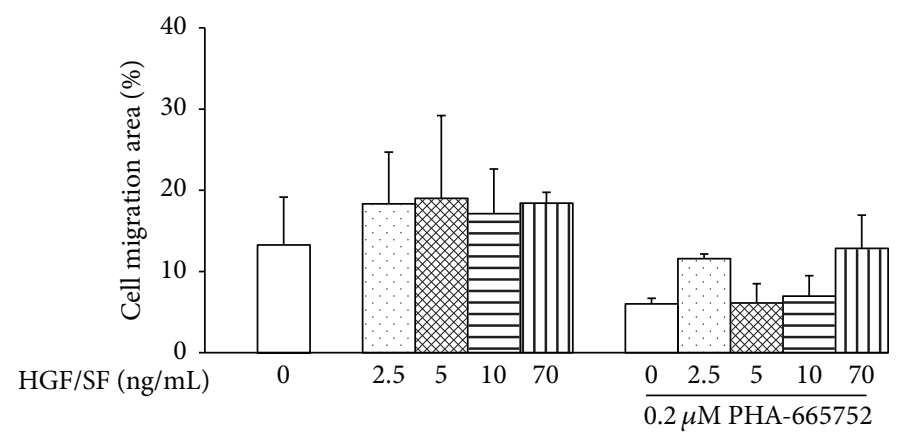

(b)
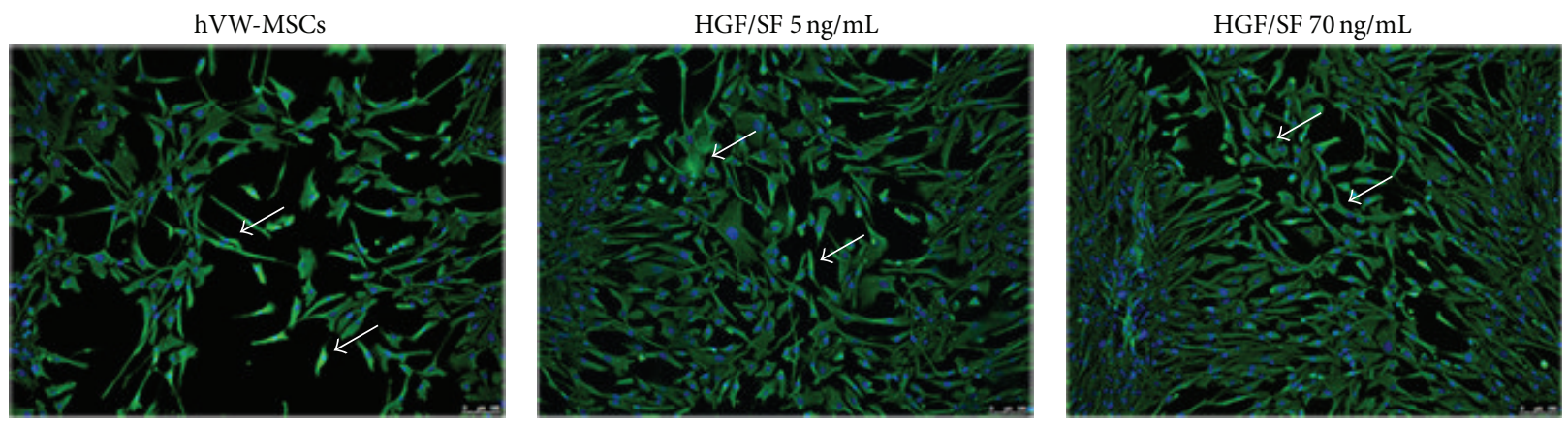

(c)

FIGURE 3: Migratory effect of HGF/SF on hVW-MSC. (a) Crystal Violet staining of hVW-MSCs migrated into the scratch area after 24 hrs of exposure to HGF/SF in comparison to the untreated hVW-MSCs; the cell migration was slackened by PHA-665752 before treatment. Wounded area is delimited by black lines. Scale bars $=50 \mu \mathrm{m}$. (b) Quantitative analysis of cell migration area in hVW-MSCs treated with HGF/SF, in presence and absence of PHA-665752. (c) Immunofluorescence staining for Vimentin revealed that the intermediate filaments Vimentin (positive (green)) was markedly stained in migrated cells (arrows) then unmigrated cells. Scale bars $=100 \mu \mathrm{m}$. The reported images are representative of three independent experiments.

becomes more evident. At 24 hrs, the number of these structures significantly decreased, except in samples treated with HGF/SF. Untreated hVW-MSCs formed very few capillarylike structures that were completely lost after $24 \mathrm{hrs}$. Human Umbilical Vein Endothelial Cells (HUVEC) were used as positive control and spontaneously formed an extensive vascular network persisting until $24 \mathrm{hrs}$ (Figure 5(a)). The number of the capillary-like structures was enhanced in all experimental conditions in comparison to untreated hVWMSCs. Although this increase was statistically significant in VEGF condition $(69 \pm 8.4$ in VEGF $50 \mathrm{ng} / \mathrm{mL}$ versus $14 \pm 9.89$ in untreated controls, $p<0.05$; Student's $t$ test), it was more pronounced after $\mathrm{HGF} / \mathrm{SF}$ stimulation (Figure 5(b)). As revealed by flow cytometry analysis, the expression of mature endothelial cell markers, such as vWF, $\mathrm{KDR}$, and CD31, was clearly promoted by HGF/SF or VEGF stimulation. In particular, HGF/SF exerted a greater effect on vWF expression (20\% higher compared to VEGF), while KDR and CD31 were almost similar in the two treatment conditions (Figure 5(c)). The flow cytometer scores for each mature endothelial cell marker have been reported in Figure 5(d). According to the scores, hVW-MSCs preconditioned with HGF/SF and VEGF underwent a high endothelial lineage commitment that was comparable to HUVEC cells. Conversely, uninduced cells kept their mesenchymal identity.

\section{Discussion}

Ischemic foot ulcers represent a serious complication of peripheral arterial disease and the surgical revascularization remains the gold standard treatment choice, even if only $60 \%$ of cases reach an effective healing after one-year 

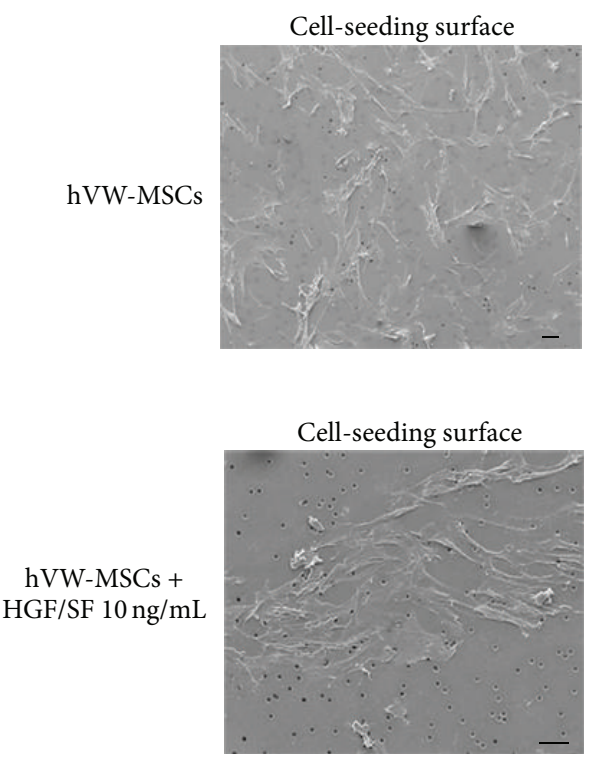

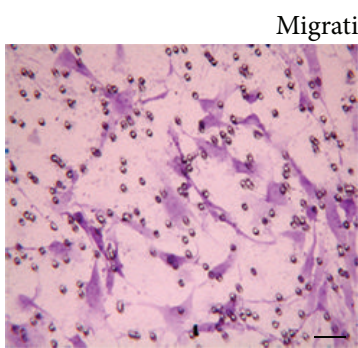

(a)
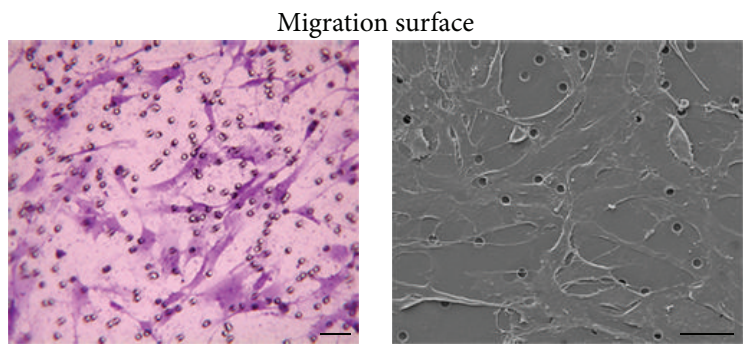

(b)

FIGURE 4: Motility effect of HGF/SF on hVW-MSCs. Representative Crystal Violet and SEM images of (a) untreated (hVW-MSCs) and (b) HGF/SF- $(10 \mathrm{ng} / \mathrm{mL})$ treated hVW-MSCs migrated through an $8 \mu \mathrm{m}$ porous membrane adhering to the migration surface. The cell-seeding surface analysis showed that the chemotactic HGF/SF facilitated the migration of many hVW-MSCs. Histological and SEM images: scale bars $=50 \mu \mathrm{m}$.

treatment $[2,25]$. Wound healing is an active and complex physiological process characterized by a cascade of events such as homeostasis, inflammation, proliferation, epithelialization, and remodeling; the interplay among many cell types, growth factors, and cytokines regulates each wound healing steps to achieve a rapidly and complete wound closure [16]; any alteration of these phases may cause an ineffective tissue repair leading to chronic wounds and their management requires a very long healing time.

Many strategies have been proposed to accelerate the wound healing process; among those, proangiogenic gene and progenitor cell therapy $[3,26]$, engineered dermis substitute implantation $[2,4]$, stem cells transplantation [9-11, 27], topical application of human platelet-derived products [1315], and growth factors such as PDGF, VEGF, TGB-beta, FGF, EGF, and GM-CFS $[16,17]$ have been developed.

In particular, HGF appears as an interesting multifaceted protein involved in the entire spectrum of the wound healing process [28]; HGF has been reported to participate to cell morphogenesis, motogenesis, mitogenesis, and tissue homeostasis, repair, and regeneration through the activation of its receptor, c-Met. Moreover, HGF is also endowed with antiapoptotic, anti-inflammatory, and antifibrotic activities [29] as well as proangiogenesis functions [30, 31].

In our study, we explored the HGF/SF effects on human stem cells to define a possible mechanism able to improve the wound healing process. In particular, we chose to investigate whether HGF/SF could positively influence the multipotent mesenchymal stem cells that reside within the vascular wall [24] and that have the potentiality to be mobilized in the injured tissue to contribute to better and faster wound healing.
HGF and its c-Met receptor are normally expressed by epithelial and endothelial cells $[32,33]$ and bone marrow mesenchymal stem cells [21-23]; here, we demonstrate that HGF and its receptor are also expressed in our cell model both at the mRNA and protein level. In particular, when hVW-MSCs cultures were exposed to HGF/SF for $24 \mathrm{hrs}$, an increase of the c-Met receptor protein levels could be detected, especially at $10 \mathrm{ng} / \mathrm{mL}$.

The HGF effects on cell proliferation are rather heterogeneous and seem to be cell and tissue dependent [21, 30, 3436]; few studies reported that HGF/SF inhibits mesenchymal stem cells proliferation through the cell cycle arrest [22, 37]. In this study, we observed that the administration of exogenous HGF/SF reduces hVW-MSC proliferation; the cell proliferation was assayed by using different techniques, that is, Alamar Blue and immunofluorescence. The proliferative response was not influenced by long (3 to 7 days) HGF/SF exposure time, whereas it was significantly increased by short time ( $6 \mathrm{hrs}$ ) at $10 \mathrm{ng} / \mathrm{mL}$ of HGF/SF. At $24 \mathrm{hrs}$ of exposure time, hVW-MSC proliferation was significantly decreased using different concentration. Even though a slight reduction of Ki-67 nuclear expression was seen at $24 \mathrm{hrs}$ of HGF/SF treatments, parallel experiments performed with the c-Met antagonist PHA demonstrated that such effect was independent of the activation of the HGF/c-MET axis. Substantially, our results demonstrate that $\mathrm{HGF} / \mathrm{SF}$ did not exert significant effects on hVW-MSC proliferation via c-Met receptor.

The migration and the repopulation of the damaged area is a necessary step to reach the complete healing of wounds. Among the multiple biological effects of HGF, the motogenic activity appears to be one of the most powerful activities as shown in a wide range of several cell types [21, 22, 30, 34]. 


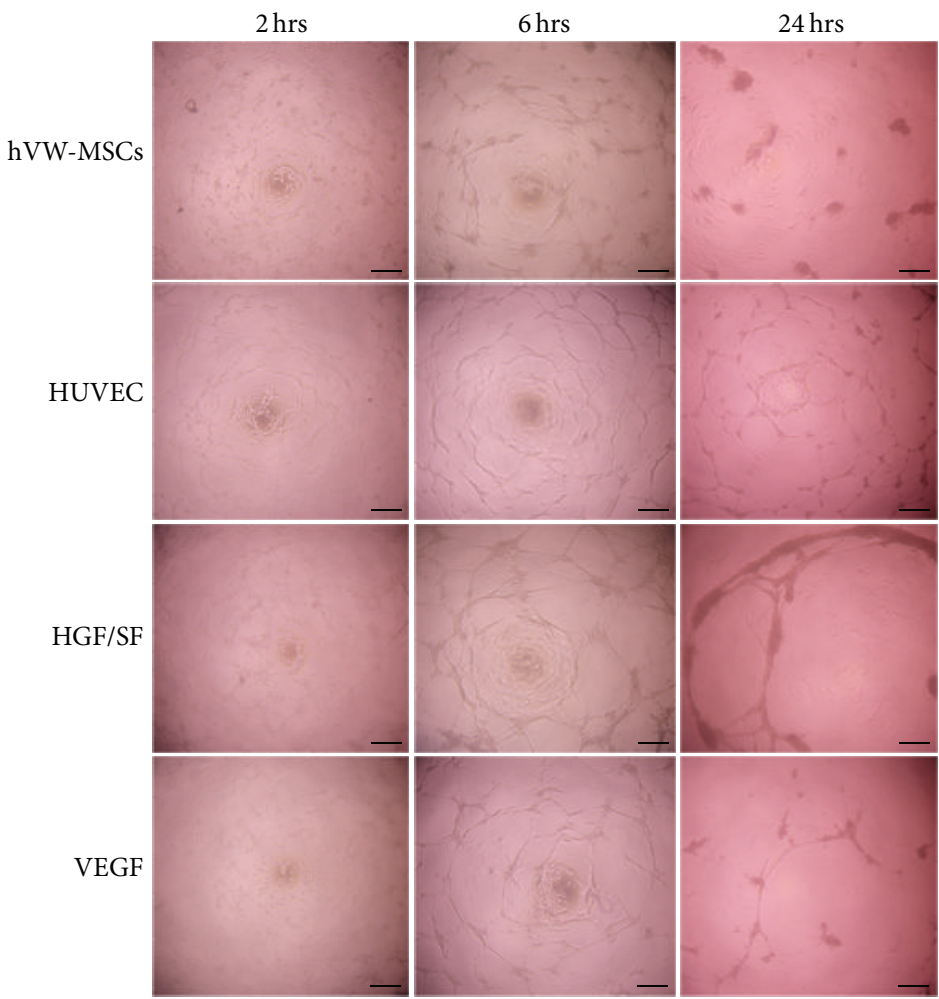

(a)

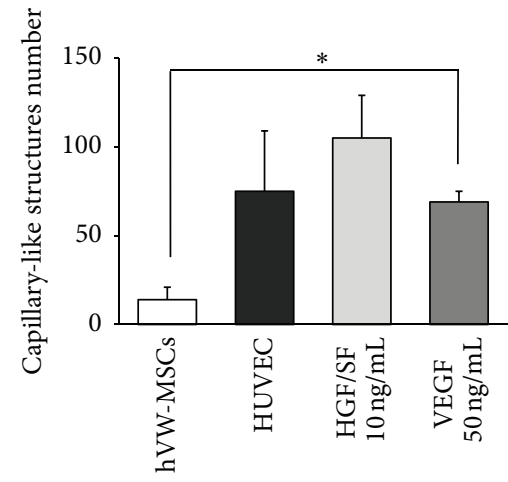

(b)

\begin{tabular}{lccc}
\hline & vWF (\%) & KDR (\%) & CD31 (\%) \\
\hline hVW-MSCs & 14.9 & 0 & 0 \\
HUVEC & 95.7 & 22.9 & 95.8 \\
hVW-MSCs HGF/SF 10 ng/mL & 95.6 & 57.1 & 93 \\
hVW-MSCs VEGF 50 ng/mL & 73.1 & 68 & 93.9 \\
\hline
\end{tabular}

(c)

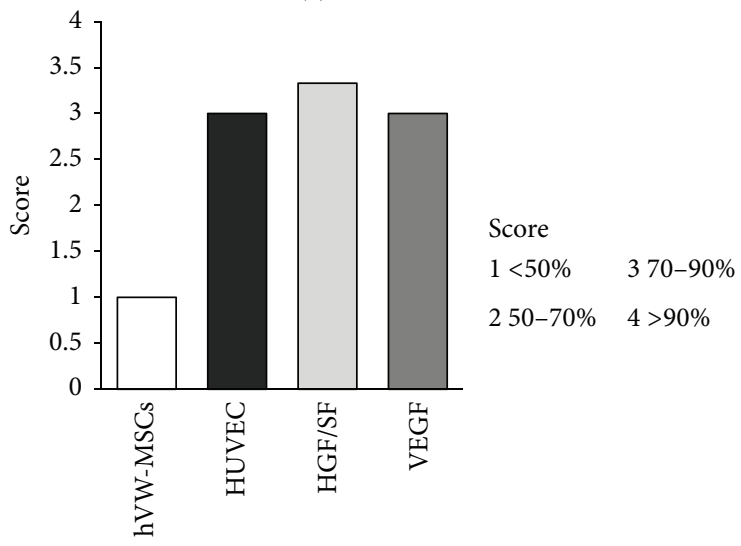

(d)

FIGURE 5: Angiogenic effect of HGF/SF on hVW-MSCs. (a) Representative in vitro tube-like formation images of HGF/SF (10 ng/mL for 7 days) and VEGF (50 ng/mL for 7 days) preconditioned hWV-MSCs as well as uninduced hWV-MSCs and HUVEC in Matrigel assay for 2, 6 , and $24 \mathrm{hrs}$ from seeding. Scale bars $=100 \mu \mathrm{m}$. (b) The quantitative analysis of the capillary-like structures revealed a higher number of hVW-MSCs exposed to HGF/SF than VEGF-treated cells. ${ }^{*} p$ value $<0.05$; Student's $t$-test. (c) Flow cytometry analysis of von Willebrand factor (vWF), KDR, and CD31 expression on untreated hVW-MSCs, HUVEC, and HGF/SF- and VEGF-treated cells. (d) The angiogenic lineage commitment was evaluated for each treatment attributing a score from 0 to 4 according to flow cytometry values.

The scratch assay is standardized, simple, and inexpensive approach used to detect this property [38]. Accordingly, we analyzed the HGF/SF effect on wounded confluent monolayers of hVW-MSCs, by using several morphological approaches including histological staining, SEM, and immunofluorescence. Our results demonstrated that different doses of HGF/SF augmented the hVW-MSC ability to migrate in the cell-free area restoring the wounded cell monolayer. Moreover, these migrating cells expressed more intensely Vimentin, an intermediate filaments marker; this feature could be related to the cytoskeleton remodeling phenomenon. The hVW-MSC migration was reduced in the presence of the c-Met receptor inhibitor, PHA-665752, even if the HGF/SF addition did not induce the complete repopulation of the wounded area. As regard the untreated hVW-MSCs, we saw spontaneous migration, contrary to literature, probably related to the autocrine secretion of endogenous HGF released by the same multipotent cells.

The chemoattractive property of HGF/SF was confirmed through the transwell migration assay. The histological and ultrastructural observations demonstrated that hVW-MSCs were able to cross the porous membrane, as attracted by HGF/SF placed at underlying bottom, and colonize the layer directly in contact with the growth factor. In the clinical context, it could be therefore speculated that a steady disposal of HGF/SF in the wound could recruit endogenous stem cells, thus promoting tissue repair.

Therapeutic angiogenesis represents another crucial event in wound healing process. VEGF is a well-established proangiogenic factor in promoting blood neovessels formation and angiogenesis [39]. In our previous studies [24, 40], VEGF has been demonstrated to be highly effective in 
promoting hVW-MSC endothelial cell lineage commitment. In that regard, also $\mathrm{HGF}$ was proposed as a powerful angiogenic promoter $[30,31,41]$ able to stimulate endothelial cell proliferation, alignment, and organization into blood vessel-like structures, although it was originally discovered as a potent growth factor for liver regeneration [29]. We assessed the HGF/SF angiogenic potential on our cell model through an in vitro 3D angiogenesis assay using Matrigel; we succeeded in demonstrating that HGF/SF pretreated hVWMSCs possess a high ability to form an extensive capillarylike network. Remarkably, the HGF/SF angiogenic effect on hVW-MSCs was comparable to that seen in the positive control (HUVEC) and with the VEGF pretreatment; following HGF/SF (10 ng/mL) stimulation, the resulting capillarylike structures persisted even at $24 \mathrm{hrs}$, suggesting that the $\mathrm{HGF} / \mathrm{SF}$ could act on the vascular integrins or adhesion molecules, therefore stabilizing the endothelial cell junctions. HGF/SF and VEGF growth factors greatly increased the expression of $\mathrm{vWF}, \mathrm{KDR}$, and CD31, typical mature endothelium markers, supporting an endothelial cell lineage commitment.

According to our data, the population of vascular mesenchymal stem cells basically expresses the HGF and its receptor, c-Met. Interestingly, the $\mathrm{HGF} / \mathrm{SF}$ addition was shown to enhance the hVW-MSC migration and motility as well as the capillary-like structures formation. These data suggest that a novel therapeutic strategy, based on the local delivery of HGF/SF and the involvement of the vascular wall-MSCs, could be developed to accelerate the healing of unresponsive vascular ulcers.

\section{Conflict of Interests}

The authors declare that there is no conflict of interests regarding the publication of this paper. Sabrina Valente is partially supported by a Grant from Octapharma AG, Lachen, Switzerland.

\section{Acknowledgment}

The authors are grateful to Sabine Noehring from Octapharma Biopharmaceuticals $\mathrm{GmbH}$, Berlin, Germany, for her constructive criticism that allowed setting up the experimental design of the study.

\section{References}

[1] N. J. M. London and R. Donnelly, "ABC of arterial and venous disease. Ulcerated lower limb," British Medical Journal, vol. 320, no. 7249, pp. 1589-1591, 2000.

[2] G. Mulder, M. Tenenhaus, and G. F. D'Souza, "Reduction of diabetic foot ulcer healing times through use of advanced treatment modalities," International Journal of Lower Extremity Wounds, vol. 13, no. 4, pp. 335-346, 2014.

[3] M. Shimamura, H. Nakagami, H. Koriyama, and R. Morishita, "Gene therapy and cell-based therapies for therapeutic angiogenesis in peripheral artery disease," BioMed Research International, vol. 2013, Article ID 186215, 8 pages, 2013.
[4] T. Lazic and V. Falanga, "Bioengineered skin constructs and their use in wound healing," Plastic and Reconstructive Surgery, vol. 127, pp. 75S-90S, 2011.

[5] H.-J. You, S.-K. Han, J.-W. Lee, and H. Chang, "Treatment of diabetic foot ulcers using cultured allogeneic keratinocytes-a pilot study," Wound Repair and Regeneration, vol. 20, no. 4, pp. 491-499, 2012.

[6] W. A. Marston, J. Hanft, P. Norwood, and R. Pollak, “The efficacy and safety of Dermagraft in improving the healing of chronic diabetic foot ulcers: results of a prospective randomized trial," Diabetes Care, vol. 26, no. 6, pp. 1701-1705, 2003.

[7] D. S. Kwon, X. Gao, Y. B. Liu et al., "Treatment with bone marrow-derived stromal cells accelerates wound healing in diabetic rats," International Wound Journal, vol. 5, no. 3, pp. 453463, 2008.

[8] Y. Wu, L. Chen, P. G. Scott, and E. E. Tredget, "Mesenchymal stem cells enhance wound healing through differentiation and angiogenesis," Stem Cells, vol. 25, no. 10, pp. 2648-2659, 2007.

[9] S.-K. Han, H.-R. Kim, and W.-K. Kim, "The treatment of diabetic foot ulcers with uncultured, processed lipoaspirate cells: a pilot study," Wound Repair and Regeneration, vol. 18, no. 4, pp. 342-348, 2010.

[10] C. Nie, D. Yang, J. Xu, Z. Si, X. Jin, and J. Zhang, "Locally administered Adipose-derived stem cells accelerate wound healing through differentiation and vasculogenesis," Cell Transplantation, vol. 20, no. 2, pp. 205-216, 2011.

[11] H. C. Lee, S. G. An, H. W. Lee et al., "Safety and effect of adipose tissue-derived stem cell implantation in patients with critical limb ischemia-a pilot study," Circulation Journal, vol. 76, no. 7, pp. 1750-1760, 2012.

[12] K. M. Lacci and A. Dardik, "Platelet-rich plasma: support for its use in wound healing," Yale Journal of Biology and Medicine, vol. 83, no. 1, pp. 1-9, 2010.

[13] V. R. Driver, J. Hanft, C. P. Fylling, and J. M. Beriou, "A prospective, randomized, controlled trial of autologous plateletrich plasma gel for the treatment of diabetic foot ulcers," Ostomy Wound Management, vol. 52, no. 6, pp. 68-74, 2006.

[14] H. S. Setta, A. Elshahat, K. Elsherbiny, K. Massoud, and I. Safe, "Platelet-rich plasma versus platelet-poor plasma in the management of chronic diabetic foot ulcers: a comparative study," International Wound Journal, vol. 8, no. 3, pp. 307-312, 2011.

[15] T. Slesaczeck, H. Paetzold, T. Nanning et al., "Autologous derived, platelet-rich plasma gel in the treatment of nonhealing diabetic foot ulcer: a case report," Therapeutic Advances in Endocrinology and Metabolism, vol. 3, no. 2, pp. 75-78, 2012.

[16] S. Enoch, J. E. Grey, and K. G. Harding, "ABC of wound healing. Recent advances and emerging treatments," British Medical Journal, vol. 332, no. 7547, pp. 962-965, 2006.

[17] S. Barrientos, H. Brem, O. Stojadinovic, and M. Tomic-Canic, "Clinical application of growth factors and cytokines in wound healing," Wound Repair and Regeneration, vol. 22, no. 5, pp. 569$578,2014$.

[18] K. Matsumoto and T. Nakamura, "Emerging multipotent aspects of hepatocyte growth factor," Journal of Biochemistry, vol. 119, no. 4, pp. 591-600, 1996.

[19] K. Okunishi, M. Dohi, K. Nakagome et al., "A novel role of hepatocyte growth factor as an immune regulator through suppressing dendritic cell function," Journal of Immunology, vol. 175, no. 7, pp. 4745-4753, 2005. 
[20] D. P. Bottaro, J. S. Rubin, D. L. Faletto et al., "Identification of the hepatocyte growth factor receptor as the c-Met proto-oncogene product," Science, vol. 251, no. 4995, pp. 802-804, 1991.

[21] S. Neuss, E. Becher, M. Wöltje, L. Tietze, and W. JahnenDechent, "Functional expression of HGF and HGF receptor/cmet in adult human mesenchymal stem cells suggests a role in cell mobilization, tissue repair, and wound healing," Stem Cells, vol. 22, no. 3, pp. 405-414, 2004.

[22] G. Forte, M. Minieri, P. Cossa et al., "Hepatocyte growth factor effects on mesenchymal stem cells: proliferation, migration, and differentiation," Stem Cells, vol. 24, no. 1, pp. 23-33, 2006.

[23] K. K. Aenlle, K. M. Curtis, B. A. Roos, and G. A. Howard, "Hepatocyte growth factor and $\mathrm{p} 38$ promote osteogenic differentiation of human mesenchymal stem cells," Molecular Endocrinology, vol. 28, no. 5, pp. 722-730, 2014.

[24] S. Valente, F. Alviano, C. Ciavarella et al., "Human cadaver multipotent stromal/stem cells isolated from arteries stored in liquid nitrogen for 5 years," Stem Cell Research \& Therapy, vol. 5, no. 1, article 8, 2014.

[25] W. J. Jeffcoate and K. G. Harding, "Diabetic foot ulcers," The Lancet, vol. 361, no. 9368, pp. 1545-1551, 2003.

[26] R. Morishita, H. Makino, M. Aoki et al., "Phase I/IIa clinical trial of therapeutic angiogenesis using hepatocyte growth factor gene transfer to treat critical limb ischemia," Arteriosclerosis, Thrombosis, and Vascular Biology, vol. 31, no. 3, pp. 713-720, 2011.

[27] E. V. Badiavas and V. Falanga, "Treatment of chronic wounds with bone marrow-derived cells," Archives of Dermatology, vol. 139, no. 4, pp. 510-516, 2003.

[28] K. Conway, P. Price, K. G. Harding, and W. G. Jiang, "The molecular and clinical impact of hepatocyte growth factor, its receptor, activators, and inhibitors in wound healing," Wound Repair and Regeneration, vol. 14, no. 1, pp. 2-10, 2006.

[29] T. Nakamura and S. Mizuno, "The discovery of Hepatocyte Growth Factor (HGF) and its significance for cell biology, life sciences and clinical medicine," Proceedings of the Japan Academy B: Physical and Biological Sciences, vol. 86, no. 6, pp. 588-610, 2010.

[30] F. Bussolino, M. F. Di Renzo, M. Ziche et al., "Hepatocyte growth factor is a potent angiogenic factor which stimulates endothelial cell motility and growth," The Journal of Cell Biology, vol. 119, no. 3, pp. 629-641, 1992.

[31] S.-I. Hayashi, R. Morishita, S. Nakamura et al., "Potential role of hepatocyte growth factor, a novel angiogenic growth factor, in peripheral arterial disease: down-regulation of HGF in response to hypoxia in vascular cells," Circulation, vol. 100, supplement 19, pp. II301-II308, 1999.

[32] M. F. Di Renzo, R. P. Narsimhan, M. Olivero et al., "Expression of the Met/HGF receptor in normal and neoplastic human tissues," Oncogene, vol. 6, no. 11, pp. 1997-2003, 1991.

[33] Y. Nakamura, R. Morishita, J. Higaki et al., "Expression of local hepatocyte growth factor system in vascular tissues," Biochemical and Biophysical Research Communications, vol. 215, no. 2, pp. 483-488, 1995.

[34] J.-M. Zahm, C. Debordeaux, B. Raby, J.-M. Klossek, N. Bonnet, and E. Puchelle, "Motogenic effect of recombinant HGF on airway epithelial cells during the in vitro wound repair of the respiratory epithelium," Journal of Cellular Physiology, vol. 185, no. 3, pp. 447-453, 2000.

[35] R. Ramanujum, Y.-L. Lin, J.-K. Liu, and S. He, "Regulatory expression of MMP-8/MMP-9 and inhibition of proliferation, migration and invasion in human lung cancer A549 cells in the presence of HGF variants," Kaohsiung Journal of Medical Sciences, vol. 29, no. 10, pp. 530-539, 2013.

[36] R. Joplin, T. Hishida, H. Tsubouchi et al., "Human intrahepatic biliary epithelial cells proliferate in vitro in response to human hepatocyte growth factor," The Journal of Clinical Investigation, vol. 90, no. 4, pp. 1284-1289, 1992.

[37] K. Chen, C. Perez-Stable, G. D’Ippolito, P. C. Schiller, B. A. Roos, and G. A. Howard, "Human bone marrow-derived stem cell proliferation is inhibited by hepatocyte growth factor via increasing the cell cycle inhibitors p53, p21 and p27," Bone, vol. 49, no. 6, pp. 1194-1204, 2011.

[38] C.-C. Liang, A. Y. Park, and J.-L. Guan, "In vitro scratch assay: a convenient and inexpensive method for analysis of cell migration in vitro," Nature Protocols, vol. 2, no. 2, pp. 329-333, 2007.

[39] K. E. Johnson and T. A. Wilgus, "Vascular endothelial growth factor and angiogenesis in the regulation of cutaneous wound repair," Advances in Wound Care, vol. 3, no. 10, pp. 647-661, 2014.

[40] G. Pasquinelli, P. L. Tazzari, C. Vaselli et al., "Thoracic aortas from multiorgan donors are suitable for obtaining resident angiogenic mesenchymal stromal cells," Stem Cells, vol. 25, no. 7, pp. 1627-1634, 2007.

[41] S. Ding, T. Merkulova-Rainon, Z. C. Han, and G. Tobelem, "HGF receptor up-regulation contributes to the angiogenic phenotype of human endothelial cells and promotes angiogenesis in vitro," Blood, vol. 101, no. 12, pp. 4816-4822, 2003. 

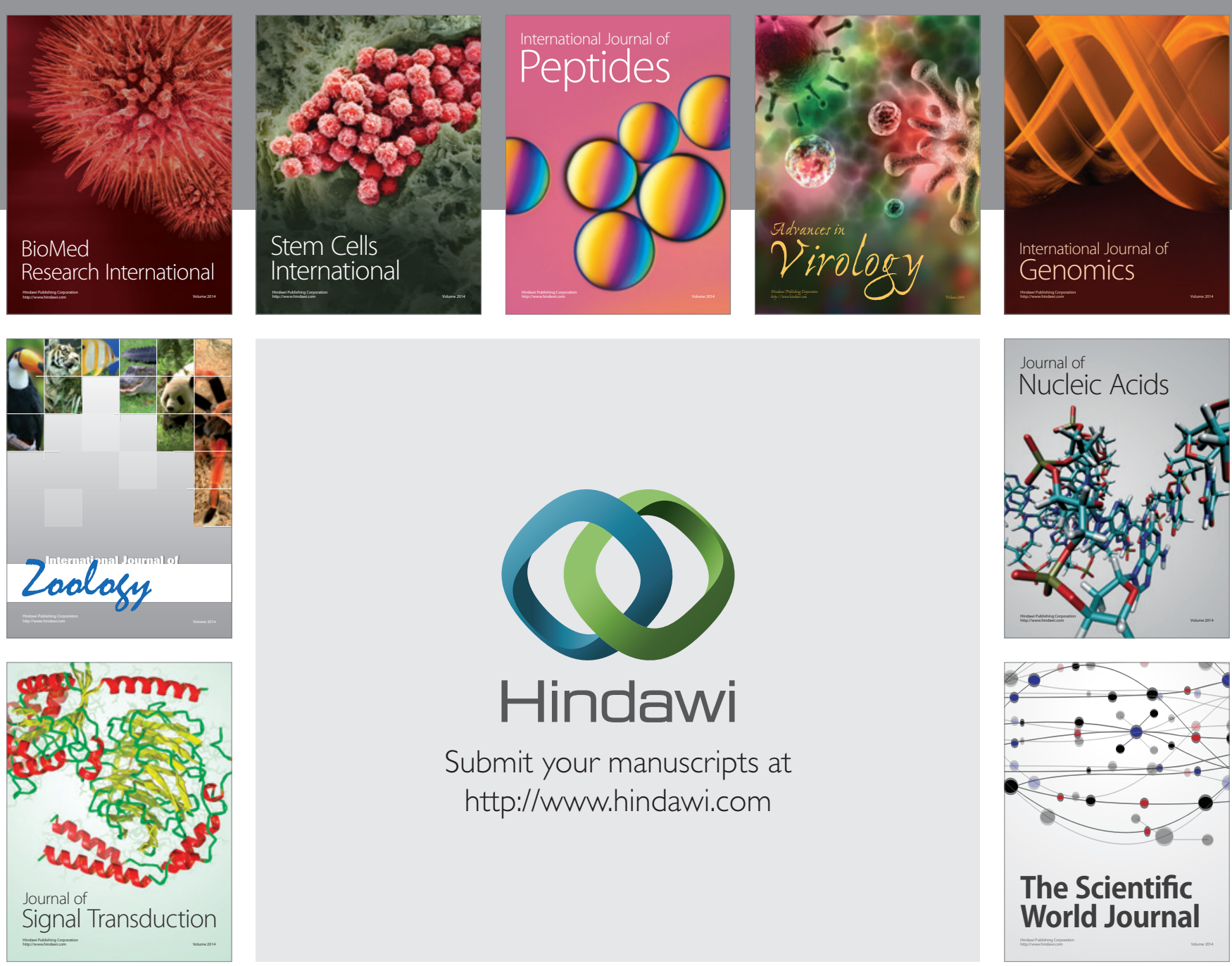

Submit your manuscripts at

http://www.hindawi.com
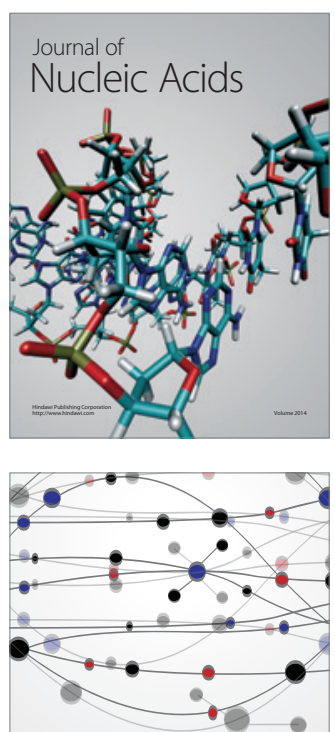

The Scientific World Journal
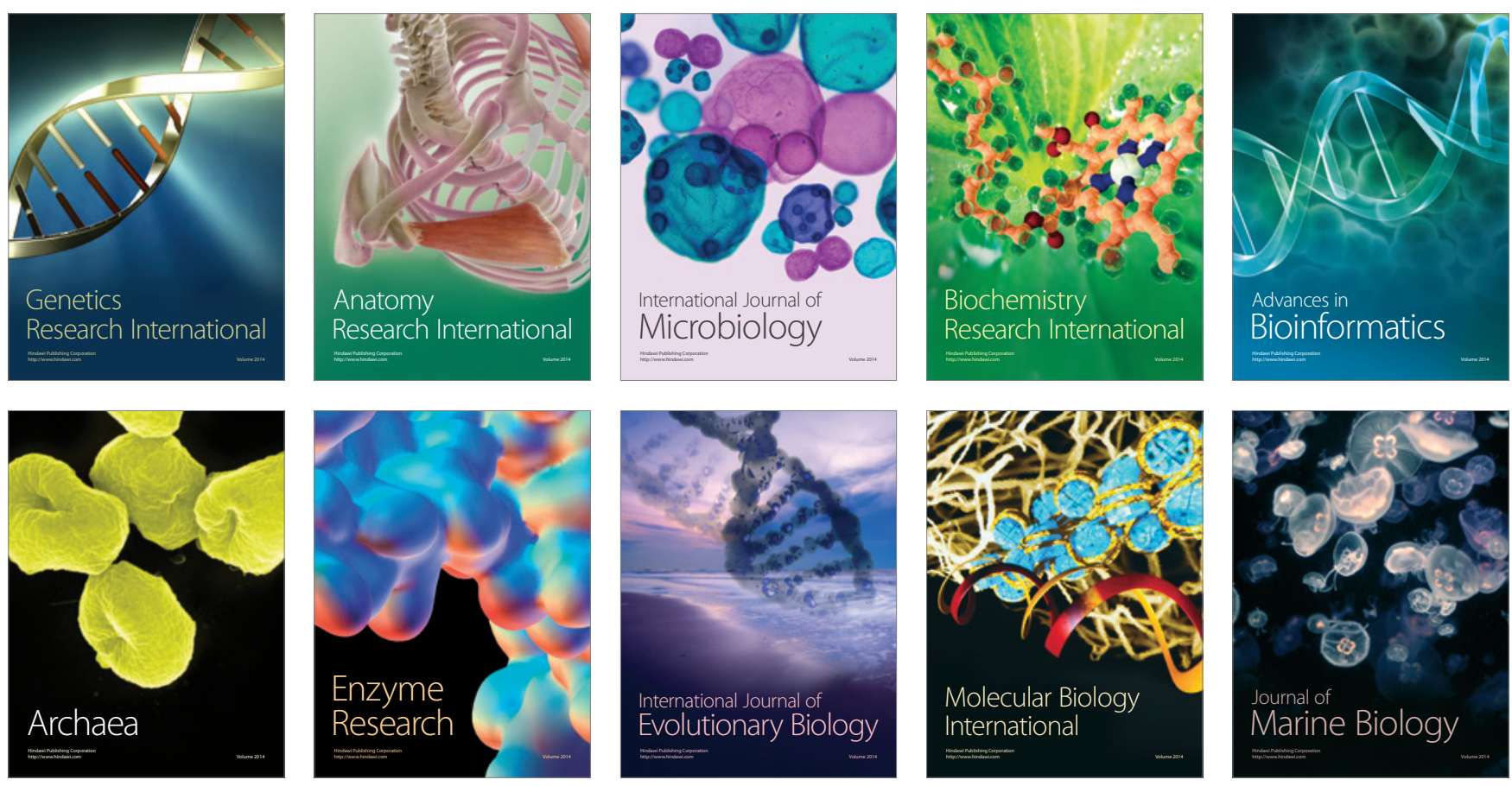